\title{
EXTENSION OF AN INEQUALITY OF H. ALZER FOR NEGATIVE POWERS
}

\author{
CHAO-PING CHEN AND FENG QI
}

Abstract. In this paper, we show that let $n$ be a natural number, then for all real numbers $r$,

$$
\frac{n}{n+1}<\left(\frac{1}{n} \sum_{i=1}^{n} i^{r} / \frac{1}{n+1} \sum_{i=1}^{n+1} i^{r}\right)^{1 / r}<1 .
$$

Both bounds are best possible. This extends a result of H. Alzer, who established this inequality for $r>0$.

In $[1,2,4,5]$, it was shown that

$$
\frac{n}{n+1}<\left(\frac{1}{n} \sum_{i=1}^{n} i^{r} / \frac{1}{n+1} \sum_{i=1}^{n+1} i^{r}\right)^{1 / r}
$$

for $r>0, n \in \mathbb{N}=:\{1,2, \ldots\}$. The lower bound is best possible. (1) is called Alzer's inequality [1].

The main purpose of this note is to provide an extension of the result given by Alzer. In order to show that the inequality (1) holds not only for $r \in(0,+\infty)$ but even for $r \in(-\infty,+\infty)$, we need the following lemma.

Lemma. Let $r \neq-1,0$ be a real number, define the function $f$ by

$$
f(x)=\frac{(x+1)^{r}}{(x+1)^{r+1}-x^{r+1}} \quad(x>0) .
$$

Then

(a) For $r \in(-\infty,-1) \cup(0,+\infty)$, the function $f$ is strictly decreasing on $(0,+\infty)$;

Received September 26, 2003.

2000 Mathematics Subject Classification. 26D15.

Key words and phrases. Inequality, power mean, Lagrange's mean value theorem, mathematical indution.

The authors were supported in part by NNSF (\#10001016) of China, SF for the Prominent Youth of Henan Province (\#0112000200), SF of Henan Innovation Talents at Universities, Doctor Fund of Jiaozuo Institute of Technology, China. 
(b) For $r \in(-1,0)$, the function $f$ is strictly increasing on $(0,+\infty)$.

Proof. Easy computation yields

$$
f^{\prime}(x)=-\frac{(x+1)^{r-1}\left[(x+1)^{r+1}-x^{r+1}-(r+1) x^{r}\right]}{\left[(x+1)^{r+1}-x^{r+1}\right]^{2}} .
$$

By Lagrange's mean value theorem, there exists at least one point $\xi \in(x, x+1)$ such that

$$
(x+1)^{r+1}-x^{r+1}=(r+1) \xi^{r}, \quad x<\xi<x+1 .
$$

Further, we have

$$
f^{\prime}(x)=-\frac{(r+1)(x+1)^{r-1}\left(\xi^{r}-x^{r}\right)}{\left[(x+1)^{r+1}-x^{r+1}\right]^{2}} .
$$

It is easy to see that for $r \in(-\infty,-1) \cup(0,+\infty), f^{\prime}(x)<0(x>0)$, and for $r \in(-1,0)$, $f^{\prime}(x)>0(x>0)$. The proof is complete.

We are now in a position to establish our result.

Theorem. Let $n$ be a natural number. Then for all real numbers $r$,

$$
\frac{n}{n+1}<\left(\frac{1}{n} \sum_{i=1}^{n} i^{r} / \frac{1}{n+1} \sum_{i=1}^{n+1} i^{r}\right)^{1 / r}<1 .
$$

Both bounds are best possible.

Proof. It was shown in [3] that for all natural numbers $n$,

$$
\frac{n}{n+1}<\frac{\sqrt[n]{n !}}{\sqrt[n+1]{(n+1) !}}<1
$$

For $r=0$, (2) can be interpreted as (3) because of

$$
\lim _{r \rightarrow 0}\left(\frac{1}{n} \sum_{i=1}^{n} i^{r} / \frac{1}{n+1} \sum_{i=1}^{n+1} i^{r}\right)^{1 / r}=\frac{\sqrt[n]{n !}}{\sqrt[n+1]{(n+1) !}} .
$$

For $r=-1$, the inequality (2) holds clearly.

For $r \in(-\infty,-1) \cup(0,+\infty)$, the left-hand inequality of (2) is equivalent to

$$
\sum_{i=1}^{n} i^{r}>\frac{n^{r+1}(n+1)^{r}}{(n+1)^{r+1}-n^{r+1}} .
$$

Clearly, the inequality (4) holds for $n=1$. Suppose (4) holds for some $n \geq 1$. Adding $(n+1)^{r}$ to the both sides of (4) leads to

$$
\sum_{i=1}^{n+1} i^{r}>\frac{(n+1)^{2 r+1}}{(n+1)^{r+1}-n^{r+1}} .
$$


By mathematical induction, it remains to show that

$$
\sum_{i=1}^{n+1} i^{r}>\frac{(n+1)^{r+1}(n+2)^{r}}{(n+2)^{r+1}-(n+1)^{r+1}} .
$$

From (5) and (6) it suffices to show that

$$
\frac{(n+1)^{r}}{(n+1)^{r+1}-n^{r+1}}>\frac{(n+2)^{r}}{(n+2)^{r+1}-(n+1)^{r+1}}
$$

which was shown in Lemma $(a)$.

For $r \in(-1,0)$, the left-hand inequality of $(2)$ is equivalent to

$$
\sum_{i=1}^{n} i^{r}<\frac{n^{r+1}(n+1)^{r}}{(n+1)^{r+1}-n^{r+1}} .
$$

Clearly, the inequality (7) holds for $n=1$. Now accepting ( 7 ) for $n \geq 1$, we try to obtain it for $n+1$. It is easy to see that the induction step can be written as

$$
\frac{(n+1)^{r}}{(n+1)^{r+1}-n^{r+1}}<\frac{(n+2)^{r}}{(n+2)^{r+1}-(n+1)^{r+1}}
$$

which was shown in Lemma $(b)$. Hence, the left-hand inequality of (2) holds for all real numbers $r$.

For $r>0$, the right-hand inequality of (2) is equivalent to

$$
\sum_{i=1}^{n} i^{r}<n(n+1)^{r}
$$

which follows obviously.

For $r<0$, the right-hand inequality of (2) is equivalent to

$$
\frac{1}{n} \sum_{i=1}^{n} i^{r}>\frac{1}{n+1} \sum_{i=1}^{n+1} i^{r} .
$$

Setting $s=-r$, then (9) can be written as

$$
\sum_{i=1}^{n} \frac{1}{i^{s}}>\frac{n}{(n+1)^{s}}
$$

which follows obviously. Hence, the right-hand inequality of (2) holds for all real numbers $r$.

It is easy to see that

$$
\begin{aligned}
& \lim _{r \rightarrow+\infty}\left(\frac{1}{n} \sum_{i=1}^{n} i^{r} / \frac{1}{n+1} \sum_{i=1}^{n+1} i^{r}\right)^{1 / r}=\frac{n}{n+1}, \\
& \lim _{r \rightarrow-\infty}\left(\frac{1}{n} \sum_{i=1}^{n} i^{r} / \frac{1}{n+1} \sum_{i=1}^{n+1} i^{r}\right)^{1 / r}=1 .
\end{aligned}
$$


Thus, the both bounds given in (2) are best possible. The proof is complete.

In view of (2), (10) and (11), it is natural to pose the following conjecture.

Conjecture. For any given natural number $n$, define the function $f$ by

$$
f(r)= \begin{cases}\left(\frac{1}{n} \sum_{i=1}^{n} i^{r} / \frac{1}{n+1} \sum_{i=1}^{n+1} i^{r}\right)^{1 / r}, & r \neq 0 \\ \frac{\sqrt[n]{n !}}{\sqrt[n+1]{(n+1) !}}, & r=0\end{cases}
$$

Then, the function $f(r)$ is strictly decreasing on $(-\infty,+\infty)$.

\section{References}

[1] H. Alzer, On an inequality of H. Minc and L. Sathre, J. Math. Anal. Appl. 179 (1993), 396-402.

[2] Ch.-P. Chen and F. Qi, Notes on proofs of Alzer's inequality, Octogon Mathematical Magazine, 11 (2003), 29-33.

[3] H. Minc and L. Sathre, Some inequalities involving $(r !)^{1 / r}$, Proc. Edinburgh Math. Soc. $14(1964 / 65), 41-46$.

[4] J. Sándor, On an inequality of Alzer, J. Math. Anal. Appl. 192 (1995), 1034-1035.

[5] J. S. Ume, An elementary proof of H. Alzer's inequality, Math. Japon. 44 (1996), 521-522.

Department of Applied Mathematics and Informatics, Jiaozuo Institute of Technology, \#142, Mid-Jiefang Road, Jiaozuo City, Henan 454000, China.

E-mail: chenchaoping@sohu.com

Department of Applied Mathematics and Informatics, Jiaozuo Institute of Technology, \#142, Mid-Jiefang Road, Jiaozuo City, Henan 454000, China.

E-mail qifeng@jzit.edu.cn or qifeng618@hotmail.com

URL: http://rgmia.vu.edu.au/qi.html 